\title{
Generation of Powerful Subterahertz Superradiance Pulses for High-Gradient Acceleration of Charged Particles
}

\author{
A. Vikharev $^{1}$, N. Ginzburg ${ }^{1,2}$, S. Kuzikov ${ }^{1}$, I. Zotova ${ }^{1}$, M.Yalandin $^{3}$ \\ ${ }^{1}$ Institute of Applied Physics RAS, Nizhny Novgorod, Russia, alvikharev@appl.sci-nnov.ru \\ ${ }^{2}$ N. I. Lobachevsky State University, Nizhny Novgorod, Russia \\ ${ }^{3}$ Institute of Electrophysics, UB RAS, Ekaterinburg, Russia
}

In currently existing conventional linear accelerators fed by klystrons operating in S-band and X-band, acceleration gradients typically not exceeded $100 \mathrm{MV} / \mathrm{m}$ [1]. This is the reason why for electrons acceleration up to high energies long and, correspondingly, cost-demanding setups are required. Substantial increase in acceleration gradient would allow for practical implementation of colliders with $\mathrm{TeV}$ power level of colliding particles; for substantial improvement of parameters and/or reducing the cost of X-ray free electron lasers currently being designed; for development of compact accelerators for radiography with sub-nanosecond synchronization and definition, for medical, industrial and other applications, etc. Correspondingly, this problem became a subject of rather intense investigation including the development of brand new concepts such as laser-plasma and beam-plasma acceleration, and also acceleration in optical nanostructures. However, investigations on improving the conventional schemes of accelerators based on electrons acceleration in hollow metallic non-superconducting ("warm") structures fed by RF radiation remain relevant. Increasing the acceleration gradient in so-called "warm" accelerators operating at room temperature is limited by effects of near-wall breakdown and pulsed thermal heating of the walls which limit the accelerating field values and deterioration of the structures surface under cyclic action of powerful microwave radiation. Despite the fact that investigations of breakdown phenomena and of walls heating have been conducted for several decades already, the only efficient method of suppressing these negative effects is considered to be the use of systems operating in the short pulse regimes when those effects have no time for settling. At that, shortening of RF pulse with simultaneous increase of its frequency, which would increase the shunt resistance considerably, would allow for relatively easy way of reaching accelerating fields of about $200 \mathrm{MV} / \mathrm{m}$ and more without exceeding threshold heat loads and surface breakdown fields. Whereas at higher frequencies the output power of RF klystrons conventionally used in accelerators decreases, the problem of design and development of high-power high-efficiency pulse sources operating in millimeter wavelength range.

In this paper we propose to use for the purpose of high-gradient particles acceleration the new class of pulsed relativistic RF generators based on the Cherenkov superradiance (SR) of high-current extended electron bunches. Currently, SR generators provide record-breaking power level in $\mathrm{mm}$ wavelength range up to $2 \mathrm{GW}$ in ultrashort pulses with a duration of 250-300 ps [2]. Investigations conducted lately show viability both of further increase of SR pulses peak power at $8-\mathrm{mm}$ wavelength band (due to coherent summation of such pulses [3,4]) and for advancement of SR generators into the short-wavelength part of the millimeter band with a power level of 50-70 MW due to using oversized electrodynamic structures [5].

In experiments on particles acceleration the experimental setup [4] consisting of two synchronized high-voltage RADAN modulator could be used. Based on the electron beam in the first section, pulsed high-frequency radiation would be generated. The accelerating structure would be positioned on the course of the electron beam in the second section. Thus, a nanosecond or subnanosecond RF radiation pulse after transmission through the quasi-optical line and reflection from the special reflector at the cathode side would feed the positioned structure in the regime of wave co-moving with the accelerated beam. Further results of PIC simulations are presented for two experimental configurations.

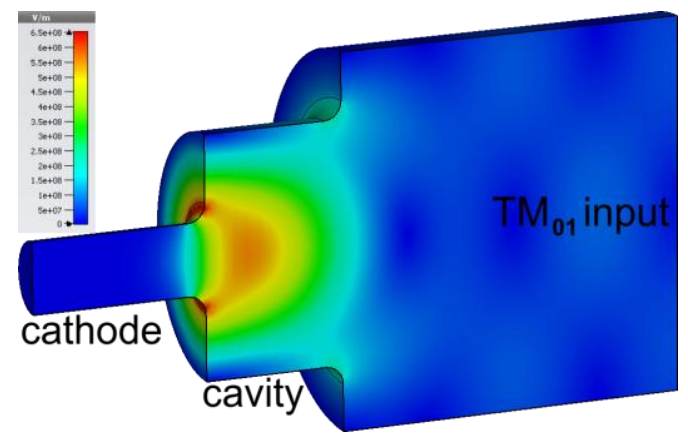

(a)

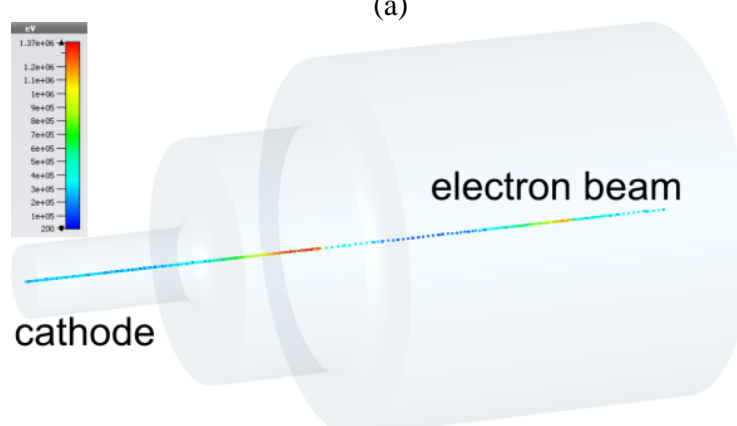

(b)

Fig. 1 (a) Geometry of a pillbox-type resonator with distribution of em field of a SR pulse. (b) The energy distribution of an electron beam with an initial energy of $300 \mathrm{keV}$ (the maximum energy of the accelerated fractions is of $1.3 \mathrm{MeV}$, the acceleration gain is of $550 \mathrm{MV} / \mathrm{m}$ ).

In the first experiments, SR pulses would be used at $38 \mathrm{GHz}$ frequency with a duration of up to $250 \mathrm{ps}$ and power of $\sim 500 \mathrm{MW}$. In this combination of parameters, acceleration in short half-wave resonator is 
of interest, which allows to obtain rather high acceleration gradient. Pillbox-type resonator at TM010 mode (see Fig. 1) with loaded quality factor of $Q=30$ at the resonance frequency is capable of providing maximum accelerating field on the axis when fed by a pulse with duration of $250 \mathrm{ps}$. According to simulations, maximum acceleration gradient reaches $550 \mathrm{MV} / \mathrm{m}$ with surface waves of up to $650 \mathrm{MV} / \mathrm{m}$. Maximum energy gain at the parameters stated would be more than $1 \mathrm{MeV}$.

It should be noted, that implementation of the described scheme at higher frequency, $140 \mathrm{GHz}$, is of unquestionable interest. At the pulse parameters (200 ps, $50 \mathrm{MW}$ ) obtained in experiments on pulse generation [5], acceleration gradient twice as high could be obtained as the parameters of the experiment to be conducted at $38 \mathrm{GHz}$ frequency. Accelerated electrons energy would be less (up to $700 \mathrm{keV}$ ), but due to smaller resonator dimensions, accelerating gradient and surface fields appear to be larger.

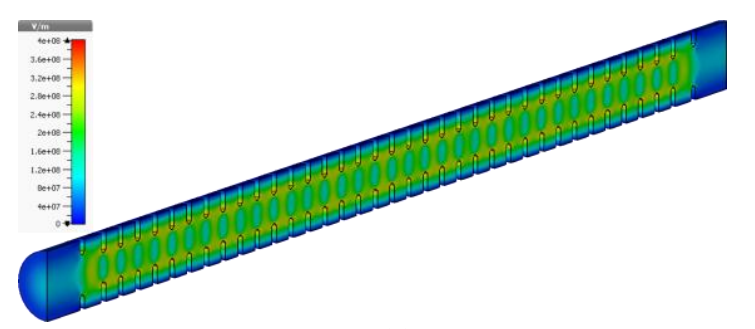

(a)

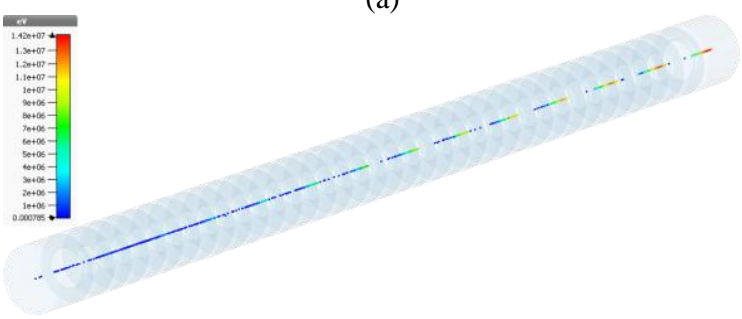

(b)

Fig. 2 (a) Geometry of an accelerating structure with distribution of em field. (b) The energy distribution of an electron beam with an initial energy of $300 \mathrm{keV}$ (the maximum energy of the accelerated fractions is of $14 \mathrm{MeV}$ ).

In the second experimental configuration, a relatively long ( $2.5 \mathrm{~ns})$ electromagnetic pulse with a power of up to $100 \mathrm{MW}$ at the $38 \mathrm{GHz}$ frequency generated by a relativistic BWO would be used for feeding of periodic hollow metallic structure with a length of 10 $\mathrm{cm}$ (see Fig. 2). Such $2 \pi / 3$ structure consists of coupled accelerating cells with a length of $3 \mathrm{~mm}$. Group velocity in the structure should be $11 \%$ of the velocity of light according to the considerations of its full feeding by a $2.5 \mathrm{~cm}$ pulse, shunt resistance of about $90 \mathrm{MOhm} / \mathrm{m}$ and the cells Q-factor $\sim 3500$. These pa- rameters are close to those of the CLIC structure at a frequency of $30 \mathrm{GHz}$ ( Rsh $=97.5 \mathrm{Mohm} / \mathrm{m}, \mathrm{Q}=$ $3900, \mathrm{Vgr} / \mathrm{c}=7 \%)$. According to simulations, averaged over the length acceleration gradient would be $220 \mathrm{MV} / \mathrm{m}$ with gained accelerated particles energy over $20 \mathrm{MeV}$. Such an experiment is of considerable interest because it simulates a so-called RF gun, which represents a source of electron beams of accelerators with higher energies (in the presence of synchronized laser providing the photoemission of the electron bunch). Implementation of such gun layout would allow for decrease of the dark current, breakdown frequency and potentially higher brightness of accelerated electron bunches comparing to conventional design.

This research was performed within the framework of the RAS Presidium project No. 10

\section{References}

1. A. Degiovanni, S. Doebert, W. Farabolini, A. Grudiev, J. Kovermann, E. Montesinos, G. Riddone, I. Syratchev, R. Wegner, W. Wuensch, A. Solodko, B. Woolley, High-gradient test results from a CLIC prototype accelerating structure: TD26CC, Proceedings of IPAC2014, Dresden, Germany, WEPME015. P. 22885-2287.

2. Rostov, V.V., Romanchenko, I.V., Pedos, M.S., Rukin, S.N., Sharypov, K.A., Shpak, V.G., Shunailov, S.A., Ul'masculov, M.R., Yalandin, M.I. Superradiant Ka-band Cherenkov oscillator with 2-GW peak power // Physics of Plasmas 2016. V. 23, No. 9. P. 093103 (1-4).

3. Ginzburg, N.S., Cross, A.W., Golovanov, A.A., Mesyats, G.A., Pedos, M.S., Phelps, A.D.R., Romanchenko, I.V. Rostov, V. V., Rukin, S.N., Sharypov, K.A., Shpak, V.G., Shunailov, S.A., Ulmaskulov, M.R., Yalandin, M.I., Zotova, I.V. Generation of electromagnetic fields of extremely high intensity by coherent summation of Cherenkov superradiance pulses// Phys. Rev. Lett. 2015. V. 115. P.114802 (1-4).

4. Mesyats, G. A., Ginzburg, N.S., Golovanov, A.A., Denisov, G. G., Romanchenko, I. V., Rostov, V.V., Sharypov, K. A., Shpak, V. G., Shunailov, S. A., Ulmaskulov, M. R., Yalandin, M. I., Zotova, I. V. //Phase-imposing initiation of Cherenkov superradiance emission by an ultrashortseed microwave pulse // Phys.Rev.Lett. 2017. V.118. P. 264801.

5. Ginzburg, N.S., Malkin, A.M., Sergeev, A.S., Zheleznov, I.V., Zotova, I V., Zaslavsky, V.Yu., Boltachev, G.Sh., Sharypov, K.A., Shunailov, S.A., Ul'masculov, M.R., Yalandin, M.I. Generation of sub-terahertz superradiance pulses based on excitation of a surface wave by relativistic electron bunches moving in oversized corrugated waveguides // Phys. Rev. Lett. 2016. V. 117. P. 204801. 